\title{
DATA ENVELOPMENT ANALYSIS (DEA) - ANÁLISE DE EFICIÊNCIA DA GESTÃO DE PORTFÓLIO DE PROJETOS DE DESENVOLVIMENTO DE PRODUTOS
}

\section{DATA ENVELOPMENT ANALYSIS (DEA) - ANALYSIS OF EFFICIENCY OF MANAGEMENT PORTFOLIO DEVELOPMENT PROJECTS PRODUCTS}

\section{Regina Rocha Morais Gonçalves}

Mestre em Administração de Empresas pela Fundação Pedro Leopoldo - FPL

E-mail: reginarmg@gmail.com (Brasil)

\section{José Edson Lara}

Doutor em Economia de 1’Empresa pelo Universitat Autònoma de Barcelona, Espanha Professor da Fundação Pedro Leopoldo - FPL

E-mail: jedson2010@ hotmail.com (Brasil)

\section{Ana Lucia Miranda Lopes}

Doutorado em Engenharia de Produção pela Universidade Federal de Santa Catarina - UFSC Coordenadora do Núcleo Estudos NESP da Universidade Federal de Minas Gerais - UFMG E-mail: analopes.ufmg@gmail.com (Brasil)

\section{Ronaldo Lamounier Locatelli}

Doutor em Economia pela University of London, Inglaterra

Professor da Fundação Pedro Leopoldo - FPL

E-mail: ronaldo.locatelli@yahoo.com.br (Brasil) 


\title{
DATA ENVELOPMENT ANALYSIS (DEA) - ANÁLISE DE EFICIÊNCIA DA \\ GESTÃO DE PORTFÓLIO DE PROJETOS DE DESENVOLVIMENTO DE PRODUTOS
}

\begin{abstract}
RESUMO
Este estudo aplicou a metodologia Data Envelopment Analysis (DEA) em uma indústria siderúrgica. Esta metodologia se baseia em modelos matemáticos não paramétricos que avalia o desempenho de cada unidade de observação com uma perspectiva multidimensional. Foi estudado um portfólio composto por 12 projetos, para os quais foram obtidos os escores de eficiência técnica e de escala. Os escores de eficiência técnica indicaram os projetos "benchmarks", comprovando a potencialidade do método. Para efeito de análises, foram aplicadas as técnicas de Data Envelopment Analysis (DEA) com o modelo BCC (Banker, Charnes e Cooper, 1984) orientado à entrada, a análise de conteúdo e o modelo NTCR (Novidade, Tecnologia, Complexidade e Ritmo). Pode-se concluir que a adoção de metodologias voltadas à gestão da eficiência de projetos é ferramenta indispensável para a competitividade da organização, motivação dos colaboradores, melhoria dos processos de gestão e redução do tempo de entrega de produtos.
\end{abstract}

Palavras-chave: Data Envelopment Analysis (DEA); Desenvolvimento de Produtos; BCC; Eficiência Técnica e de Escala.

\section{DATA ENVELOPMENT ANALYSIS (DEA) - ANALYSIS OF EFFICIENCY OF MANAGEMENT PORTFOLIO DEVELOPMENT PROJECTS PRODUCTS}

\begin{abstract}
This study applied the methodology Data Envelopment Analysis (DEA) in Steel Industry. The DEA is based on non-parametric mathematical models. It evaluates the performance of each unit of observation with a multidimensional perspective. The study used a portfolio of 12 projects for which were obtained the scores of technical and scale efficiency. The technical efficiency scores indicated the "benchmarks" projects, demonstrating the potential of DEA. For purposes of analysis, the search used the Data Envelopment Analysis with BCC (Banker, Charnes, Cooper, 1984) inputoriented model; content analysis and the NTCR model (News, Technology, Complexity and Rhythm). It can be concluded that the adoption of methodologies aimed at efficiency projects management is an essential tool for competitiveness of the organization, motivating employees, improving management processes and reducing time for product delivery
\end{abstract}

Keywords: DEA; BCC; Product Development Management; Technical Efficiency and Scale. 


\section{INTRODUÇÃO}

A capacidade das empresas introduzirem novos produtos no mercado assume a cada dia maior relevância para a sua sobrevivência. Para manterem competitivas, as empresas estão elaborando suas estratégias baseadas na inovação. Schumpeter (1949) apresenta a inovação como impulso fundamental para a permanência e expansão das organizações no mercado, apontando-a como o principal motor do desenvolvimento capitalista e fonte de lucro empresarial. A inovação pode se manifestar no novo desenho do produto, no novo processo de produção, na nova abordagem de marketing ou nos novos métodos de trabalho ou treinamentos.

A situação apresentada cria uma interdependência entre as áreas da organização, pois requerem informações e cooperação entre os agentes dos diversos departamentos funcionais, como Marketing, Desenvolvimento de Produtos, Pesquisa e Desenvolvimento (P\&D), Produção e Qualidade, além de uma constante interação com o cliente, com o objetivo de obter a convergência para os objetivos estratégicos da empresa.

Neste sentido, Clark e Wheelwright (1993) ressaltam que a integração multifuncional é fundamental para uma performance diferenciada no processo de desenvolvimento de um produto, quando se consideram as dimensões financeira, mercadológica e técnica.

Este contexto traz como solução para a indústria a Gestão de Desenvolvimento de Produtos (GDP). Entretanto, conforme Rozenfeld et al. (2006) é constatado que o sucesso da organização no desenvolvimento de novos produtos não é garantido pela genialidade ou criatividade dos profissionais de $\mathrm{P} \& \mathrm{D}$, ou pelo número de recursos alocados aos projetos, além das habilidades técnicas, dependem das práticas e dos modelos de gestão adotados.

Embora muitos estudos tenham sido publicados nos últimos anos, entre outros podem ser considerados, Pinto e Slevin (1988), Clark e Wheelwright (1993), Toledo et. Al. (2006), Rozenfeld et al. (2006), Shenhar e Dvir (2010). No Brasil, ainda, existem poucas publicações que apontam as causas para o sucesso e ou o fracasso dos projetos, e nenhum acordo de entendimento foi alcançado até o momento.

Shenhar e Dvir (2010) identificaram cinco dimensões de sucesso dos projetos: Eficiência do Projeto, Impacto no cliente; Impacto na Equipe, Sucesso Comercial e Direto e Preparação para o futuro.

Neste contexto esta pesquisa aplicou a metodologia Data Envelopment Analysis (DEA) em uma indústria siderúrgica. Esta metodologia se baseia em modelos matemáticos não paramétricos

Revista de Gestão e Projetos - GeP, São Paulo, v. 4, n. 3, p 119-140, set./dez. 2013. 
que avalia o desempenho de cada unidade de observação com uma perspectiva multidimensional. Proposto inicialmente em 1978 por Charnes, Cooper e Rhodes o modelo que assumiu retorno constante de escala (CCR), Charnes, Cooper, Rhodes, (1978), Mendes, Lopes e Banker (2012), Athanasios (2012) . Algumas evoluções ocorreram, e em 1984 Banker, Charnes e Cooper desenvolveram o modelo que permitia retorno variável de escala (BCC), (BANKER, CHARNES e COOPER, 1984). Ambos os modelos podem ser classificados como orientados a insumos ou a produtos, dependendo das definições de metas das unidades analisadas.

A DEA foi utilizada como uma estrutura para analisar a eficiência técnica e de escala da gestão de projetos de desenvolvimento de produtos. Foi estudado um portfólio composto por 12 projetos, para os quais foram obtidos os escores de eficiência técnica e de escala. Os escores de eficiência técnica indicaram os projetos "benchmarks", comprovando a potencialidade do método. Para efeito de análises, foram aplicadas as técnicas: DEA com o modelo BCC (Banker, Charnes, Cooper - VRS - Variable Returns to Scale), a análise de conteúdo e o modelo NTCR (Novidade, Tecnologia, Complexidade e Ritmo). Pode-se concluir que a adoção de metodologias voltadas à gestão da eficiência de projetos é ferramenta indispensável para a competitividade da organização, motivação dos colaboradores, melhoria dos processos de gestão e redução do tempo de entrega de produtos.

Para apresentar os resultados das soluções propostas, este artigo está organizado em cinco seções. A primeira se destina a apresentar o tema em estudo, sua contextualização e as proposições da pesquisa. A segunda apresenta as três perspectivas do referencial teórico a estratégia e a gestão de desenvolvimento de produtos; avaliação de desempenho de projetos de desenvolvimento de produtos, e o modelo da Data Envelopment Analysis. A terceira seção aborda a metodologia de pesquisa utilizada e a quarta mostra a análise dos resultados, sob a luz do referencial teórico adotado. A quinta seção apresenta um conjunto de conclusões e recomendações, para se alcançar a completude da pesquisa.

\section{REFERENCIAL TEÓRICO}

O referencial teórico desta pesquisa baseou-se em três perspectivas: A Estratégia e a Gestão de Desenvolvimento de Produtos; Avaliação de Desempenho de Projetos de Desenvolvimento de Produtos, e o modelo da Data Envelopment Analysis (DEA).

Revista de Gestão e Projetos - GeP, São Paulo, v. 4, n. 3, p 119-140, set./dez. 2013. 


\subsection{A ESTRATÉGIA E A GESTÃO DO DESENVOLVIMENTO DE PRODUTOS}

Na Figura 1, Clark e Wheelwright (1993) fornecem um modelo de referência expandido, em relação aos modelos tradicionais, adiciona dois pontos focais ao pré-projeto: objetivos do desenvolvimento e um plano de projeto agregado - onde a estratégia tecnológica e a de produto/mercado podem ser discutidas e integradas. Estas atividades de pré-projeto fornecem um caminho para os gerentes tratarem questões e políticas entre projetos, e para definir limites nos projetos individuais.

Para Clark e Wheelwright (1993) a estratégia de desenvolvimento de novos produtos deve suportar as duas dimensões: tecnologia e mercado conforme a Figura 1 A estratégia tecnológica orienta as atividades nos processos de aquisição, desenvolvimento, e aplicação de tecnologia que gere vantagem competitiva. A estratégia de mercado respalda a definição dos tipos de produtos que farão parte do portfólio, principais clientes e a seleção de estratégias para manter a fidelidade dos mesmos. Assim, considera-se que a elaboração da estratégia seja importante, mas, normalmente, não é suficiente para garantir o sucesso da organização. É imprescindível que a empresa execute uma avaliação mais completa do mix de projetos que farão parte do seu plano agregado de projetos, ou seja, do seu portfólio.

Clark e Wheelwright (1993) destacam, ainda, que a integração multifuncional pode assegurar um desempenho superior nas dimensões de custo, tempo e qualidade, na medida em que a criação de novos produtos constitui um processo multidisciplinar. Este conceito também é destacado por Kerzner (2006), o qual coloca como imprescindível a integração da gestão de projetos com os demais processos da organização, entre os processos a serem integrados, são destacados: gestão da qualidade, de mudanças, de riscos, do planejamento estratégico para projetos e de engenharia simultânea.

Para pautar as estratégias das organizações em inovação, tal como têm recomendado Schumpeter (1949), Porter (1993), Skarzynnski e Gibson (2008), Tidd, Bessant e Pavitt (2008), Trot (2012), entre outros, as empresas estão melhorando a capacidade de criar novos produtos, em ciclos cada vez mais curtos, associado com a maior exigência dos consumidores, tornando a gestão de desenvolvimento de novos produtos imprescindível para alavancar a competitividade da empresa.

Revista de Gestão e Projetos - GeP, São Paulo, v. 4, n. 3, p 119-140, set./dez. 2013. 
Figura 1 - Estrutura para desenvolvimento da Estratégia

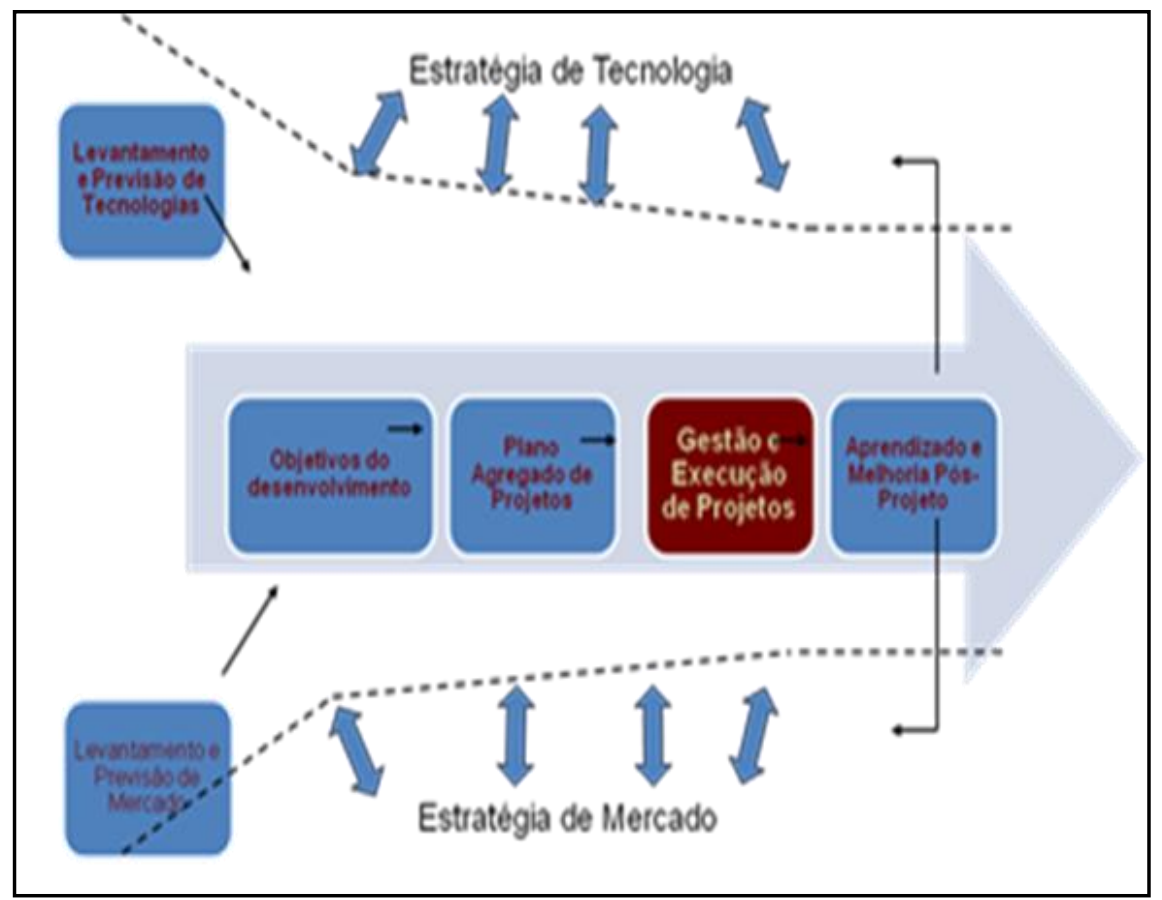

Fonte: Adaptado de CLARK; WHEELWRIGHT(1993).

A gestão do desenvolvimento de produtos, (GDP), abrange o estudo de métodos e técnicas para desenvolvimento de novos produtos e a melhoria da qualidade de produtos existentes (ROZENFELD et al., 2006). Esse autor destaca ainda que, o sucesso das empresas no desenvolvimento de produtos não é garantido pela capacidade técnica de seus profissionais e nem pelo número de recursos alocados ao projeto, mas é essencial considerar as práticas e modelos de gestão adotadas.

\subsection{AVALIAÇÃO DE DESEMPENHO DE PROJETOS DE DESENVOLVIMENTO DE PRODUTOS}

Shenhar e Dvir (2010) propuseram que o sucesso do projeto e do produto não deve ser separado porque a maioria dos projetos é parte da gestão estratégica da empresa, e por isto devem ser avaliados com base em suas contribuições para os resultados do negócio, e não apenas pela habilidade da equipe de gestão cumprir com as metas de tempo, escopo e orçamento. A Equipe do projeto precisa se envolver com os resultados que esse trará para a organização.

Revista de Gestão e Projetos - GeP, São Paulo, v. 4, n. 3, p 119-140, set./dez. 2013. 
Estes autores identificaram cinco dimensões do sucesso de projetos, para as quais existe um conjunto de variáveis que podem ser medidas conforme a relação com o tempo do projeto. A primeira dimensão, eficiência do projeto (ou o cumprimento das metas planejadas) representa as medidas de curto prazo, avaliando as metas de prazo, orçamento, resultados e outras eficiências. Entretanto estas medidas não garantem que o produto originário do desenvolvimento do projeto terá sucesso e beneficiará a empresa no longo prazo, (SHENHAR E DVIR, 2010).

A segunda dimensão, impacto no cliente, representa os envolvidos no projeto (stakeholders). É nesta dimensão que ocorre a análise da aderência dos requisitos em atendimento ao cliente, conformidade às especificações técnicas, benefícios para o cliente, para o cliente, extensão de uso, satisfação e lealdade do cliente e reconhecimento da marca.

A terceira dimensão, impacto na equipe, representa como o projeto está afetando a equipe e seus membros. Os pontos importantes medidos por esta dimensão são satisfação e moral da equipe, desenvolvimento de habilidades, crescimento e retenção dos membros da equipe e baixo stress.

A quarta dimensão, sucesso comercial, reflete o impacto direto e imediato que o projeto teve na empresa. Em relação ao negócio são avaliados os níveis de vendas, rendas e lucros, assim como os fluxos de caixa e outras medidas financeiras.

A quinta, e última dimensão, preparação para o futuro, trata dos benefícios de longo prazo do projeto. Essa dimensão auxilia a organização a preparar sua infraestrutura para o futuro e ainda a como deverão ser criadas as novas oportunidades. Estes preparam para o futuro novos processos organizacionais, bem como as competências técnicas e organizacionais adicionais. As oportunidades que serão criadas geralmente estão relacionadas com a criação de novos mercados, criação de uma nova linha de produtos ou o desenvolvimento de uma nova tecnologia.

Devido ao fato de que os projetos são únicos e individuais, gerentes e executivos precisam entender a peculiaridade de que para cada tipo de projeto, é necessário que a organização seja adequada para a execução correta do mesmo. Para atender esta necessidade, Shenhar e Dvir (2010) propuseram o modelo diamante NTCR, o qual é uma estrutura que os gerentes podem utilizar quando tomam decisões sobre projetos e como eles devem ser gerenciados. Essa estrutura possui quatro dimensões, que contemplam através de três a quatro níveis, aspectos em relação à Novidade, Complexidade, Tecnologia e Ritmo.

Na dimensão Novidade do produto, deve ser entendido o quão novo é o produto para o mercado.e para os seus usuários em potencial. Na dimensão de Tecnologia, segundo Shenhar e Dvir (2010), o principal fator de análise está relacionado ao grau de incerteza tecnológica. Eles

Revista de Gestão e Projetos - GeP, São Paulo, v. 4, n. 3, p 119-140, set./dez. 2013. 
relacionam a dimensão de Complexidade do Projeto com o escopo do sistema, de forma que é possível utilizar uma estrutura hierárquica de sistemas e subsistemas, os quais podem ser considerados os pacotes de trabalho da estrutura analítica do projeto, conforme usado por Rozenes (2004). A dimensão de Ritmo está relacionada à urgência, e as suas consequências quando as metas de tempo não são cumpridas, segundo Shenhar e Dvir (2010). Neste contexto, os projetos vem sendo estudados sob os mais amplos matizes, como relatam autores, como Moutinho, Kniess, Maccari ( 2013) e Napolitano e Rabechini Júnior (2012).

\subsection{DATA ENVELOPMENT ANALYSIS (DEA)}

Charnes, Cooper, Rhodes (1978) desenvolveram o modelo (CCR) de programação matemática para avaliação empírica da eficiência relativa de unidades tomadoras de decisão (DMUs) com base nas quantidades observadas de insumos e produtos para um grupo similar de DMUs operando, globalmente, sob retornos constantes à escala.

DMU - Decison Making Unit - são compreendidas como as unidades tomadoras de decisão sobre avaliação, e podem ser uma empresa, um departamento, um segmento de negócio, um portfólio, um projeto, ou outras.

Banker, Charnes e Cooper (1984) (BCC) relacionaram a DEA com a estimativa de fronteiras de produção eficiente estendendo o modelo de retornos constantes a escala (CRS) para retornos variáveis a escala (VRS).

A metodologia Data Envelopment Analysis (DEA) baseia-se em modelos matemáticos não paramétricos, ou seja, esta não utiliza inferências estatísticas, ou medidas de tendência central, testes de coeficientes ou formalizações de análises de regressão. A DEA permite medir a eficiência sem quaisquer suposições sobre a forma funcional da função de produção ou os pesos para entradas e saídas (FERREIRA e GOMES, 2009), e Krivonozhko, Førsund e Lychev, 2012.

Para simplificar a visualização, a Figura 2, considera-se uma entrada e uma saída. A fronteira de eficiência é a melhor prática para o conjunto de dados observados. Para cada DMU a análise DEA fornece:

- Um valor de eficiência para cada DMU

- Um conjunto de referência de eficiência

- Uma meta para cada DMU ineficiente, indicando o quanto deve ser reduzida a entrada ou expandida a saída para torná-la eficiente.

Revista de Gestão e Projetos - GeP, São Paulo, v. 4, n. 3, p 119-140, set./dez. 2013. 
Figura 2 - DEA - Orientação

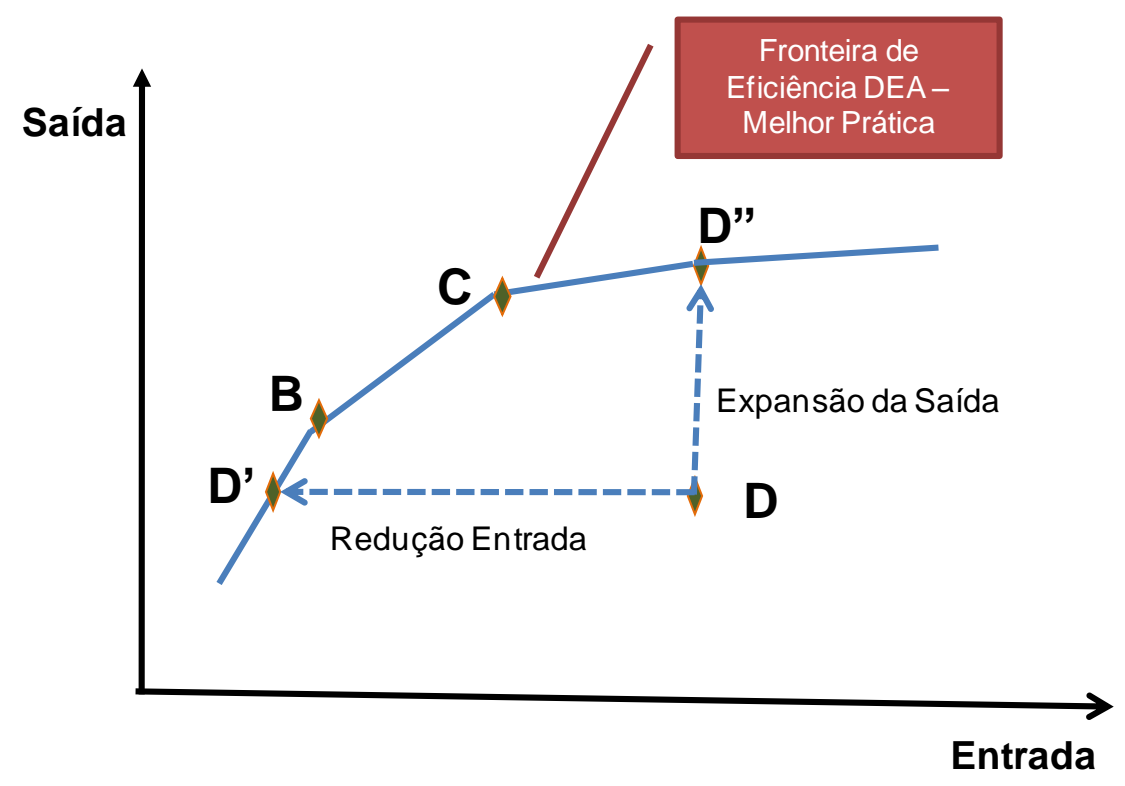

Fonte: (ZHU e COOK, 2008, pag.26)

Conforme Figura 2, a quando uma DMU, no caso a DMU D é ineficiente, existe um número de caminhos diferentes para movê-la para a fronteira de eficiência. Duas direções são fundamentais: orientada a entrada e orientada a saída. Zhu e Cook (2008) mostram que o modelo orientado a entrada permite que a entrada (insumos) seja reduzida para produzir uma saída fixada (produtos); enquanto que o modelo orientado a saída permitirá incrementar a saída (produção), mantendo a entrada (recursos) fixa.

\section{METODOLOGIA}

Quanto aos fins segundo a classificação apresentada por Collis e Hussey (2005) esta pesquisa é de caráter descritivo e exploratório. Quanto aos meios, foi utilizado um estudo de caso que contemplou as abordagens quantitativa e qualitativa.

Para a análise dos resultados foi utilizada a metodologia de data envelopment analysis (DEA) associada à análise de conteúdo e ao modelo NTCR (Novidade, Tecnologia, Complexidade e Ritmo).

Revista de Gestão e Projetos - GeP, São Paulo, v. 4, n. 3, p 119-140, set./dez. 2013. 
O estudo de caso foi realizado em uma indústria siderúrgica com base em Belo Horizonte e que está integrada a outras duas unidades no Brasil. A empresa pesquisada será denominada nesse artigo como Siderúrgica Alfa.

Para a execução do modelo DEA foram consideradas as seguintes variáveis e suas respectivas definições:

- Duração do Projeto (em Dias): é a data de início real menos a data de término real.

- Custo do Projeto (em Reais - R\$): é o valor dos custos diretos envolvidos no Projeto, ou seja, o custo realizado.

- Número de Produtos Entregues: é o número de produtos desenvolvidos com a execução do projeto.

- Toneladas Vendidas: é a quantidade de toneladas envolvidas nas solicitações do cliente para a data planejada de disponibilização do produto pelo Projeto.

Avaliou-se que o mais adequado em função da aderência da aplicabilidade para o cálculo da eficiência técnica do conjunto de projetos em análise é o Modelo BCC (VRS - retornos variáveis de escala) porque este não assume proporcionalidade entre entradas e saídas.

O Modelo BCC orientado a insumo foi adotado porque o objetivo do estudo é avaliar o quanto é possível diminuir o prazo de entrega dos produtos de cada projeto, mantendo os mesmos equipes/recursos envolvidos. Outro fato em que nessa pesquisa se recomenda a adoção do Modelo BCC vem do fato de se poder lidar com eficiências de escala, ou seja, um projeto eficiente não precisa necessariamente ter a máxima relação entre entradas e saídas.

O software selecionado para executar o método DEA desta pesquisa foi o DEA Frontier, e está disponível em www.deafrontier.com.

\section{RESULTADOS OBTIDOS}

Os resultados obtidos foram agregados em três tópicos: a empresa estudada, a aplicação da Data Envelopment Analysis (DEA) e a análise dos resultados da pesquisa. 


\subsection{DESCRIÇÃO DA SIDERÚRGICA ALFA}

A Siderúrgica Alfa tem cumprido o seu foco no mercado nacional e se confirmado como líder no mercado de aços planos, no qual tem se diferenciado a partir do desenvolvimento de produtos com alta tecnologia e da incorporação de uma estrutura de atendimento e prestação de serviços integrada. O Sistema possui capacidade instalada de produção anual de 9,5 milhões de toneladas de aço bruto, e detém 59\% do mercado interno, além de ser líder no fornecimento de aço para segmentos estratégicos da economia brasileira, como a indústria automotiva, de tubos de grande diâmetro e eletroeletrônica.

\subsection{APLICAÇÃO DA DATA ENVELOPMENT ANALYSIS (DEA)}

Conforme as orientações disponíveis para o uso do software DEA Frontier, os dados correspondentes às colunas de variáveis de entrada (duração do projeto e custo realizado) e de variáveis de saída (Número de Produtos Entregues e Oportunidades (Toneladas Vendidas)) do Quadro 1 foram utilizados com o objetivo de: calcular a eficiência técnica e de escala dos projetos do Portfólio analisado, além de identificar quais são o(s) projeto(s) benchmark(s) do Portfólio analisado.

Conforme apresentado no Quadro 2 foram obtidos os escores de eficiência técnica de cada Projeto analisado. Foram identificadas duas DMUs eficientes, a 1 e a 11, por terem atingido o valor máximo igual a 1. Estas são as DMUs benchmarks. Conforme resumido no Quadro 2, para cada DMU considerada ineficiente, estão relacionadas as DMUs referência (benchmarks). 


\begin{tabular}{|c|c|c|c|c|c|c|c|c|}
\hline \multicolumn{5}{|c|}{ DESENVOLVIMENTO DE PRODUTOS - TMCP } & \multicolumn{2}{|c|}{ Entrada } & \multicolumn{2}{|c|}{ Saída } \\
\hline $\begin{array}{c}\text { Programa } \\
\text { / Grupos }\end{array}$ & Aréas / Parceiros Envolvidos & DMU & Produtos & Projetos & $\begin{array}{c}\text { Duração } \\
\text { do } \\
\text { Projeto }\end{array}$ & Custo (R\$̦) & $\begin{array}{l}\text { Número de } \\
\text { Produtos } \\
\text { gerados }\end{array}$ & $\begin{array}{c}\text { Oportunidad } \\
\text { es Ton. = } \\
\text { (geradas - } \\
\text { perdidas) }\end{array}$ \\
\hline \multirow{3}{*}{ 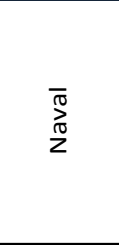 } & \multirow{3}{*}{ 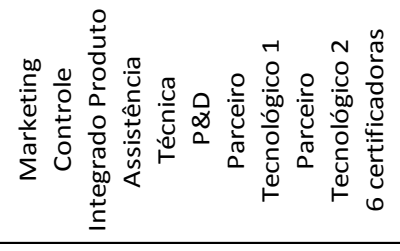 } & 1 & Naval $1,2,3,4,5,6$ & Projeto_Naval 1 & 180 & 473.650 & 6 & 15.000 \\
\hline & & 2 & Naval 7,8,9 & Projeto_Naval 2 & 180 & 473.650 & 3 & 9.000 \\
\hline & & 3 & Naval 10 & Projeto_Naval 3 & 242 & 622.450 & 1 & 8.000 \\
\hline \multirow{4}{*}{ 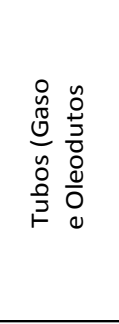 } & \multirow{4}{*}{ 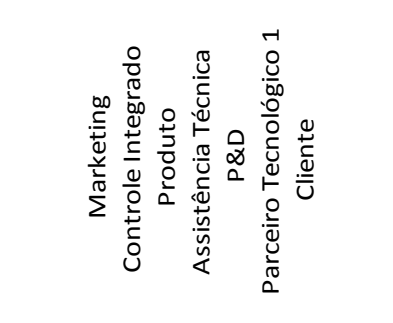 } & 4 & Tubo 1 & Projeto_Tubo 1 & 395 & 736.000 & 1 & 0 \\
\hline & & 5 & Tubo 2 & Projeto_Tubo 2 & 395 & 736.000 & 1 & 5.000 \\
\hline & & 6 & Tubo 3 & Projeto_Tubo 3 & 395 & 736.000 & 1 & 7.000 \\
\hline & & 7 & Tubo 4 & Projeto_Tubo 4 & 395 & 736.000 & 1 & 7.000 \\
\hline \multirow{5}{*}{ 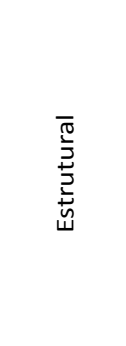 } & \multirow{5}{*}{ 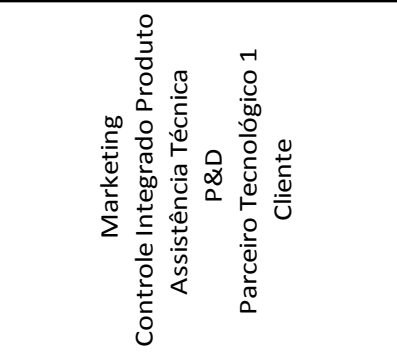 } & 8 & Estrutural 1 & Projeto_Estrutural 1 & 152 & 298.600 & 1 & 8.000 \\
\hline & & 9 & Estrutural 2 & Projeto_Estrutural 2 & 152 & 298.600 & 1 & 8.500 \\
\hline & & 10 & Estrutural 3 & Projeto_Estrutural 3 & 181 & 350.800 & 1 & 7.800 \\
\hline & & 11 & Estrutural 4 & Projeto_Estrutural 4 & 120 & 241.000 & 1 & 7.850 \\
\hline & & 12 & Estrutural 5, 6 & Projeto_Estrutural 5 & 183 & 354.400 & 2 & 6.000 \\
\hline
\end{tabular}

Quadro 1 - Portfólio de Projetos de Desenvolvimento de Novos Produtos (DNP) Fonte: Dados fornecidos pela Empresa Alfa

A execução do Modelo BCC orientado a insumo apontou as DMU's 2, 3, 4, 5, 6, 7, 8, 9, 10 e 12 como ineficientes, conforme o Quadro 2, mas cada uma apresentou um grau diferente de ineficiência. Para torná-las eficientes é necessário que cada DMU seja projetada através de uma linha que liga a origem à fronteira. Ao projetar esta linha, pode ser constatado que cada DMU ineficiente estará à distância das DMUs eficientes. Por exemplo, a DMU 2, estará entre as DMUs consideradas eficientes 1 e 11 .

Revista de Gestão e Projetos - GeP, São Paulo, v. 4, n. 3, p 119-140, set./dez. 2013. 


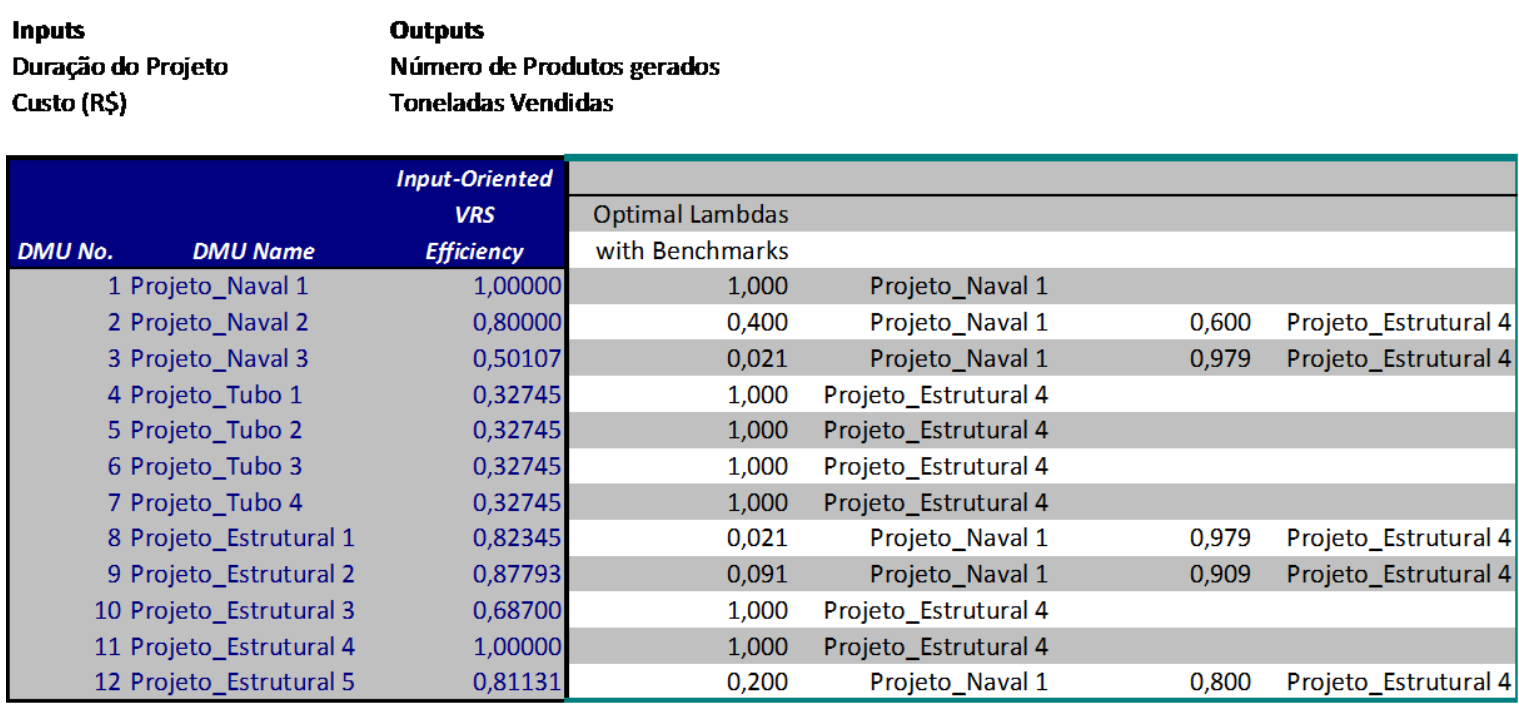

Quadro 2 - Execução do modelo BCC

Fonte: Planilha de Execução DEA-FRONTIER

Inicialmente para identificar os projetos eficientes utilizou-se o modelo BCC orientado a insumo.

Nesse modelo foi aplicada a restrição de convexidade onde, conforme definido em Zhu e Cook (2008):

$\sum_{k=1}^{n} \lambda k \square \square \square \square \square \square \square($ restrição de convexidade), onde $\lambda \mathrm{k} \geq 0$ para todo $\mathrm{k}$

A qual possibilitou a obtenção de medidas de eficiência no paradigma do modelo BCC (retornos variáveis de escala - VRS).

Para o cálculo da eficiência de escala foi necessário executar o modelo CCR para o cálculo dos escores de eficiência com retorno constante. Foi retirada a restrição de convexidade, e aplicada à execução do modelo CCR orientado a insumo.

O Quadro 3 apresenta os escores de eficiência técnica encontrados com a execução do modelo CCR orientado a insumo para o portfólio analisado.

Conforme a análise do Quadro 3 identificou-se duas DMUs que obtiveram eficiência máxima, por terem atingido o valor máximo igual a 1 , foram os Projetos 1 e 11, pertencentes ao Programa de Projetos Naval e Estrutural respectivamente. Ressalta-se que os Projetos 1 e 11 também obtiveram eficiência técnica máxima para o modelo $\mathrm{BCC}$, o que nos leva a comprovar que todo projeto eficiente no modelo CCR também será no modelo BCC, FERREIRA e GOMES, (2009).

Revista de Gestão e Projetos - GeP, São Paulo, v. 4, n. 3, p 119-140, set./dez. 2013. 


\begin{tabular}{|c|c|c|c|c|c|c|c|}
\hline $\begin{array}{l}\text { Inputs } \\
\text { Duraçāo do Projeto } \\
\text { Custo (RS) }\end{array}$ & \multicolumn{7}{|c|}{$\begin{array}{l}\text { Outputs } \\
\text { Número de Produtos gerados } \\
\text { Tonebadas Vendidas }\end{array}$} \\
\hline & Input-Oriented & & & & & & \\
\hline & CRS & Sum of & & Optimal Lambdas & & & \\
\hline DMU Name & Efficiency & lambdas & RTS & with Benchmarks & & & \\
\hline 1 Projeto_Naval 1 & 1,00000 & 1,000 & Constant & 1,000 & Projeto_Naval 1 & & \\
\hline 2 Projeto_Naval 2 & 0,60000 & 0,600 & Increasing & 0,600 & Projeto_Naval 1 & & \\
\hline 3 Projeto_Naval 3 & 0,40498 & 0,570 & Increasing & 0,493 & Projeto_Naval 1 & 0,078 & Projeto_Estrutural 4 \\
\hline 4 Projeto_Tubo 1 & 0,10726 & 0,167 & Increasing & 0,167 & Projeto_Naval 1 & & \\
\hline 5 Projeto_Tubo 2 & 0,21015 & 0,556 & Increasing & 0,089 & Projeto_Naval 1 & 0,467 & Projeto_Estrutural 4 \\
\hline 6 Projeto_Tubo 3 & 0,29246 & 0,868 & Increasing & 0,026 & Projeto_Naval 1 & 0,841 & Projeto_Estrutural 4 \\
\hline 7 Projeto_Tubo 4 & 0,29246 & 0,868 & Increasing & 0,026 & Projeto_Naval 1 & 0,841 & Projeto_Estrutural 4 \\
\hline 8 Projeto_Estrutural 1 & 0,82252 & 1,019 & Decreasing & 1,019 & Projeto_Estrutural 4 & & \\
\hline 9 Projeto_Estrutural 2 & 0,87393 & 1,083 & Decreasing & 1,083 & Projeto_Estrutural 4 & & \\
\hline 10 Projeto_Estrutural 3 & 0,68268 & 0,992 & Increasing & 0,002 & Projeto_Naval 1 & 0,991 & Projeto_Estrutural 4 \\
\hline 11 Projeto Estrutural 4 & 1,00000 & 1,000 & Constant & 1,000 & Projeto Estrutural 4 & & \\
\hline 12 Projeto_Estrutural 5 & 0,53097 & 0,489 & Increasing & 0,302 & Projeto_Naval 1 & 0,187 & Projeto_Estrutural 4 \\
\hline
\end{tabular}

Quadro 3- Execução do modelo DEA - CCR

Fonte: Planilha de Execução DEA-FRONTIER

Com as eficiências técnicas calculadas no Modelo BCC e no Modelo CCR segue-se ao cálculo do índice de eficiência de escala, conforme o Quadro 4.

Conforme o Quadro 4 as DMUs 1 e 11, projetos benchmarks, estão operando em escala ótima.

\begin{tabular}{|c|c|c|c|c|}
\hline Inputs & & Outputs & & \\
\hline \multicolumn{2}{|c|}{ Duração do Projeto } & \multicolumn{2}{|c|}{ Número de Produtos gerados } & \\
\hline \multicolumn{2}{|l|}{ Custo (R\$) } & \multicolumn{2}{|l|}{ Toneladas Vendidas } & \\
\hline & & $\begin{array}{l}\text { Input-Oriented } \\
\text { CRS }\end{array}$ & $\begin{array}{c}\text { Input-Oriented } \\
\text { VRS }\end{array}$ & Scale \\
\hline DMU No. & DMU Name & $\mathrm{CCR}$ & $B C C$ & Efficiency \\
\hline & Projeto_Naval 1 & 1,00 & 1,00 & 1,0000000 \\
\hline 2 & Projeto_Naval 2 & 0,60 & 0,80 & 0,7500000 \\
\hline 3 & Projeto_Naval 3 & 0,40 & 0,50 & 0,8082290 \\
\hline 4 & Projeto_Tubo 1 & 0,11 & 0,33 & 0,3275588 \\
\hline 5 & Projeto_Tubo 2 & 0,21 & 0,33 & 0,6417837 \\
\hline 6 & Projeto_Tubo 3 & 0,29 & 0,33 & 0,8931635 \\
\hline 7 & Projeto_Tubo 4 & 0,29 & 0,33 & 0,8931635 \\
\hline 8 & Projeto_Estrutural 1 & 0,82 & 0,82 & 0,9988788 \\
\hline 9 & Projeto_Estrutural 2 & 0,87 & 0,88 & 0,9954431 \\
\hline 10 & Projeto_Estrutural 3 & 0,68 & 0,69 & 0,9937155 \\
\hline 11 & Projeto_Estrutural 4 & 1,00 & 1,00 & 1,0000000 \\
\hline 12 & Projeto_Estrutural 5 & 0,53 & 0,81 & 0,6544521 \\
\hline
\end{tabular}

Quadro 4 - Eficiência de Escala do Portfólio de Projetos Fonte: Dados da Pesquisa

Revista de Gestão e Projetos - GeP, São Paulo, v. 4, n. 3, p 119-140, set./dez. 2013. 


\subsection{ANÁLISE DOS RESULTADOS}

A aplicação da Data Envelopment Analysis (DEA) para o cálculo da eficiência de projetos, para a identificação de projetos benchmarks e para o cálculo da eficiência de escala foi comprovada para o portfólio de projetos analisado, comprovando o potencial do método.

No Portfólio de projetos estudado, conforme o Quadro 4 pode-se perceber que $80 \%$ apresentam ineficiência técnica e de escala.

Dentre os fatores críticos de sucesso, foram identificadas as práticas descritas na sequência, que contribuíram para o sucesso dos Projetos Naval 1 e Estrutural 4, os quais foram considerados os benchmarks do portfólio analisado:

- Existência de um processo de desenvolvimento de produto bem definido, desde a ideia até o lançamento;

- Trabalho em equipes multifuncionais;

- Integração da equipe de desenvolvimento de produtos com seus clientes e fornecedores.

- Habilidades na realização das atividades de pré-desenvolvimento do projeto, tais como: geração de ideias, análise de viabilidade técnica, mercadológica e financeira;

- Capacidade de captar as necessidades dos clientes;

- Sinergia entre o produto proposto e os recursos, habilidades e vantagens (técnicas ou mercadológicas) pré-existentes na empresa;

- O líder do projeto possuía as habilidades técnicas, gerenciais e de relacionamento interpessoal necessário à gestão de conflitos e problemas;

- Todas as áreas envolvidas no PDP foram integradas através de processos para viabilizar a execução das atividades com otimização do tempo;

- Para execução do projeto foi formada uma equipe com pessoas de diferentes áreas/departamentos que participavam integralmente ou parcialmente dela, foi nomeado um líder/gerente do projeto e as pessoas envolvidas respondiam tanto ao gerente/chefe dessas áreas/departamentos como ao gerente/líder do projeto (estrutura matricial).

Outro ponto destacado como prática de sucesso, relaciona-se com a integração de um projeto a determinado programa de desenvolvimento. Um dos requisitos está relacionado com a homologação do produto, nesse momento são avaliados os envolvidos nos testes, bem como se

Revista de Gestão e Projetos - GeP, São Paulo, v. 4, n. 3, p 119-140, set./dez. 2013. 
existem órgãos externos de certificação, além de analisar se os produtos poderão ser certificados/homologados em conjunto. No planejamento dos testes são identificadas as possibilidades de reduzir o prazo de homologação dos produtos para todos os projetos interdependentes do portfólio. Nessa análise, o Gestor ressaltou que no caso do Programa Naval, considerando os Projetos Naval 1 e Naval 2, pela primeira vez na história da companhia, ocorreu a homologação de 9 produtos, com a participação de 6 entidades certificadoras navais simultaneamente. Essa integração de processos e produtos permitiu diminuir o prazo de entrega do Projeto Naval 2 em 7 (sete) meses, e ainda antecipar o atendimento de duas oportunidades que geraram a entrega de 1.000 toneladas de aço.

Os Gestores de Desenvolvimento de Novos Produtos, referente ao Projeto da DMU 1, ressaltaram a importância dada ao compartilhamento de informações e a necessidade de integração de todas as áreas da Organização no desenvolvimento dos mesmos. Para os entrevistados, a integração garantiu organizar, sistematizar e padronizar as informações advindas das diferentes áreas funcionais da empresa. Outro destaque da integração está relacionado com o aumento significativo na assertividade, na competitividade, velocidade e rentabilidade das novas soluções desenvolvidas em produtos.

Nos projetos do Programa Naval, no qual se percebe maior integração, segundo entrevista do seu gestor, essa possibilitou entregar soluções com maior cumprimento de escopo, prazo e qualidade. Estes resultados geraram novas oportunidades para a empresa, além de uma maior satisfação do cliente. Resultados estes que foram traduzidos em eficiência técnica máxima com a execução do algoritmo DEA.

Os cenários apresentados pela empresa têm utilizado como ferramenta de integração e execução de suas estratégias a gestão de projetos, a qual pode ser desenvolvida na cultura da organização independente da estrutura organizacional, onde, gestão de projetos é uma cultura, e nunca um conjunto de normas ou procedimentos (KERZNER, 2006). Sob este ponto de vista, é constatado, que a cultura de gestão de projetos está em desenvolvimento na organização, e esta pode ser uma das causas pelas quais os projetos do portfólio apresentam índices tão diferentes de eficiência técnica quando analisados utilizando o modelo data envelopment analysis (DEA). Uma das ações recomendadas para que estes índices possam alcançar todo o portfólio é a criação de um escritório de projetos, (KERZNER, 2006), com o objetivo de centralizar e integrar todos os projetos da organização, estabelecer padrões e metodologia de gestão de projetos, e ainda treinar e capacitar todos os líderes de projetos, além de realizar a coleta de lições aprendidas para disseminar e introduzir as boas práticas dos projetos benchmarks nos demais projetos da empresa. 
Foi verificado que a empresa possui um processo formal para o desenvolvimento de novos produtos, em acordo com (CLARK E WHEELWRIGHT, 1993) e (CHENG, 2000). A empresa Alfa tem os seus processos e metodologia de gestão de desenvolvimento de novos produtos baseados nas melhores práticas do (PMBOK, 2008), e o processo de desenvolvimento de novos produtos com as etapas recomendadas por Rozenfeld et al. (2006), que divide o PDP em três macro processos: prédesenvolvimento, desenvolvimento e pós-desenvolvimento. Através dos processos existe uma integração de toda a cadeia entre marketing, vendas, desenvolvimento e qualidade.

Nas entrevistas foi identificada a integração dos processos de gestão de projetos, gestão da qualidade e de mudanças, os quais são três dos cinco processos utilizados pelas empresas que já atingiram um nível de excelência (KERZNER, 2006); que são: gestão de projetos, gestão da qualidade, gerenciamento de riscos e gestão de mudanças e de engenharia simultânea.

Os entrevistados concordam que existe um relacionamento entre o grau de integração e os indicadores de desempenho da organização, enumerando vários resultados que foram alcançados provenientes de um nível satisfatório de integração, tais como, aumento da produtividade, redução no tempo de desenvolvimento, antecipação da solução dos problemas, cumprimento das datas de entrega, aumento no valor agregado dos produtos, padronização dos processos, integração dos processos, velocidade da informação e facilidade de acesso à informação. Esses resultados foram identificados nos Projetos "Benchmark".

Os escores de eficiência técnica alcançados pelos projetos identificados como benchmarks resgataram (CLARK E FUJIMOTO, 1991) e (CLARK E WHEELWRIGHT, 1993) para afirmar que a integração entre os diversos departamentos no processo de gestão de desenvolvimento de produtos aumenta a flexibilidade diante das mudanças inesperadas e a visão integrada do projeto. Esses resultados estabelecem práticas a serem implantadas para os projetos que apresentaram ineficiência técnica e de escala (ROZENES, 2004).

\section{CONCLUSÕES E RECOMENDAÇÕES}

Este estudo permitiu calcular os escores de eficiência técnica e de escala dos projetos de um determinado Portfólio utilizando a DEA. Nesse caso foi identificado que os modelos matemáticos não paramétricos da Data Envelopment Analysis (DEA) atenderam plenamente à demanda de aplicação prática, sem perder o rigor da análise científica.

Revista de Gestão e Projetos - GeP, São Paulo, v. 4, n. 3, p 119-140, set./dez. 2013. 
Conforme Shenhar e Dvir (2010), os projetos diferem de várias maneiras e um modelo não serve para todos. Os gestores de projetos de sucesso foram capazes de se adaptar ao ambiente, à tarefa e à meta, e não simplesmente se ativeram a um conjunto de regras. Esta nova abordagem adaptativa do gerenciamento de projetos exige que existam modelos para a gestão do desenvolvimento de produtos, porém, para cada projeto deve ser analisado o conjunto de melhores práticas aplicáveis.

Para contribuir com a identificação das melhores práticas a Data Envelopment Analysis (DEA) apresentou-se como um método adequado porque permite identificar os projetos "benchmark" do portfólio, através do cálculo da eficiência técnica. Os resultados obtidos permitem ao gestor do portfólio analisar e identificar pontos de melhorias que podem ser aplicados aos projetos com desvios, a fim de melhorar os resultados de um determinado conjunto de projetos.

Devido ao destaque da integração multifuncional para os projetos percebidos e comprovados de sucesso, foram estudados os mecanismos utilizados pela empresa nestes. Dentre estes mecanismos, devem ser ressaltados os processos de gestão de projetos, de mudanças, de riscos e da qualidade.

Devido à importância da DEA enquanto estrutura de conhecimento capaz de organizar grupos de variáveis de entrada e saída que explicam o fenômeno, considera-se de bom alvitre a aplicabilidade do modelo como recurso na operacionalização e predição de variáveis.

Comprovados os benefícios do modelo para os resultados das organizações, cabe a academia desenvolver e divulgar a aplicabilidade da DEA como proposta de uma metodologia para apoiar gestores em suas decisões nas organizações.

A pesquisa encontrou algumas limitações para a sua plena execução. Nesse estudo devido à inexistência de uma Gestão Integrada de Portfólio que contemple todos os projetos da organização, na Siderúrgica Alfa foi necessário utilizar uma amostra reduzida. A seleção da amostra de projetos ficou restrita aos que tinham disponíveis os dados necessários ao processamento do algoritmo DEA, limitando o número de DMUs a 12 projetos.

Outra limitação desse estudo se refere à questão de se tratar de um estudo de caso específico de uma única organização. Isso restringe as considerações e resultados, os quais devem ser analisados de forma restrita ao contexto, não podendo ser assim generalizados. A ausência de outros estudos similares para que pudesse haver um referencial comparativo é outra limitação a ser considerada.

O desenvolvimento de um modelo de referência poderá contribuir para selecionar melhor os projetos a executar, bem como aplicar os recursos para aqueles que trarão maior resultado. Esse 
modelo de referência poderá auxiliar aos gestores a antecipar a solução de problemas, reduzirem o prazo e o custo de lançamento de novos produtos, e consequentemente, viabilizar a maximização da inovação.

\section{REFERÊNCIAS}

Athanasios, Valiakos. A genetic algorithm approach to efficiency assessments with common weights, in: Proceedings of the 10th International Conference on DEA, August 2012, Natal, Brazil.

Baker, B. N., Murphy, D. C. e Fisher, D. Factors Affecting Project Success. IN Cleland, D. I. \& King, W. R. Systems analysis and Project management. New York: McGraw Hill, 1983

Banker, R. D.; Charnes, A.; Cooper, W.; Some Models for Estimating Technical and Scale Inefficiencies, in Data Envelopment Analysis. Management Science, Vol. 30, No. 9, pp. 10781092, Set. 1984.

Banker, R. D.; Cooper, W., Seiford,L.; Thrall, R.M.; Zhu, J.; Returns to scale in different DEA models. European Journal of Operational Research 154 (2004) 345-362.

Charnes, A.; Cooper, W.; Rhodes; E. Measuring the efficiency of decision making units. European Journal of Operational Research 2, 1978; 429-444.

Charnes, A.; Cooper, W.; Lewin; A.Y., Seiford,L. M. Data Envelopment Analysis: Theory, Methodology and Application. 3a. Ed. Massachussetts, USA, 1997. 513p.

Cheng, L.C. Caracterização da Gestão de desenvolvimento do produto: delineando o seu contorno e dimensões básicas, in II Congresso Brasileiro de Gestão de Desenvolvimento de Produto. São Carlos, SP. 2000.

Cooper, R. G; Edgett, S. J; Kleinschmidt, E. J. Portfolio Management in New Product Development: Lessons from the Leaders - I. Research Technology Management, v.40(5); p.1628. 1997. 
Clark, K., Fujimoto, T. Product development performance: strategy, organization and management in the world auto industry. Boston: Harvard Business School Press, 1991.

Clark, K., Wheelright, S. C. Managing New Product and Process Development: Text and Cases. New York: Fee Press, 1993.

Collis, J.; Hussey, R. Pesquisa em administração. Porto Alegre: Bookman, 2005.

Ferreira, C. M. C.; Gomes, A. P. Introdução à Análise Envoltória de Dados: Teoria, Modelos e Aplicações. Viçosa, MG: Ed. UFV, 2009.

Griffin, A. Page, A. Success Measurement Project: Recommended Measures for Product Development Success and Failure. Journal of Product Innovation Management, v. 13; p.478-496. 1996.

Griffin, A. Hauser, J. R. Integrating R\&D and Marketing: A Review and Analysis of the Literature. Journal of Product Innovation Management, v. 13; p. 191-215. 1996.

Griffin, A. PDMA Research on New Product Development Practices: Updating Trends and Benchmarking Best Practices. Journal of Product Innovation Management, v.14; p. 429- 458. 1997.

Kerzner, Harold. Gestão de Projetos: as melhores práticas. $2^{\mathrm{a}}$ Edição - Porto Alegre: Bookman, 2006. 824p.

Krivonozhko, Vladimir E.; Førsund, Finn R. Lyche, Andey V. Measurement of returns to scale using a non-radial DEA model, in: Proceedings of the 10th International Conference on DEA, August 2012, Natal, Brazil.

Mendes, Marco; Lopes, Ana Lucia Miranda; Banker, Rajiv. An effectiveness analysis of different techniques for development of IT software projects, in Proceedings of the 10th International Conference on DEA, August 2012, Natal, Brazil.

Moutinho, José A. Kniess, Claudia T. Maccari. Emerson A. A Influência da Gestão de Projetos na Estratégia de Transferências Voluntárias de Recursos da União para Municípios Brasileiros: O Caso de uma Prefeitura de Médio Porte. Revista Ibero-Americana de Estratégia. v. 12, n. 1 (2013).

Napolitano, Domingos M. e Rabechini Júnior, R. Gestão de Risco e Desempenho de Projetos Complexos: O Grid das Competências. Revista Gestão \& Tecnologia. v. 12, n. 3, p. 287-310, set./dez. 2012. 
Pinto, J. K, Slevin, D. P. Critical Success Factors Across the Project Live Cycle, in International Journal Project Management, 1986.

Pinto, J. K, Slevin, D. P. Project Success Definitions and Measurement Techniques, in International Journal Project Management, 1988.

PMBOK, 4 ${ }^{\mathrm{a}}$ Edição - A Guide to The Project Management Body of Knowledge PMI, Project Management Institute, 14 Campus Boulevard, Newtown Square, Pennsylvania: Boulevard, 2008

PMBOK, $2^{\text {a }}$ Edição - The Standard for Portfolio Management. PMI, Project Management Institute, 14 Campus Boulevard, Newtown Square, Pennsylvania: Boulevard, 2008

Porter, M. E. Vantagem Competitiva das Nações. Rio de Janeiro, Editora Campos, 1993, 512p.

Rozenes, S. Multidimensional project control system. Coventry University, PhD Thesis. (2004)

Rozenes, S., Vitner, G.; Spraggett, S. MPCS: Multidimensional Project Control System. International Journal Project Management, No. 22, 2004.

Rozenfeld, H.et al. Forcellini, F. A.; Amaral, D.C.; Toledo, J.C.; Silva, S.L.; Alliprandini, D.H; Scalice, R.K. (2006). Gestão de Desenvolvimento de Produtos: uma referência para melhoria do processo. São Paulo: 542p, Editora Saraiva.

Schumpeter, Joseph Alois. Theory of economic development: one inquiry into profits, capital, credit, interested and the business cycle. Cambridge: Harvard University, 1949. 225p.

Shenhar, Aaron.; Dvir, Dov. Reinventando o Gerenciamento de Projetos: A abordagem diamante ao crescimento e inovação bem-sucedidos. São Paulo, MBooks do Brasil, 2010. 260p.

Skarzynski, Peter; Gibson, Rowan. Inovação Prioridade $\mathrm{N}^{\circ}$ 1: O caminho para as transformações nas organizações. Rio de Janeiro, Elsevier, 2008.

Tidd, J.; Bessant, J.; Pavitt, K. Gestão da Inovação. Porto Alegre, Bookman, 2008.

Toledo, J. C.; Silva, S. L.; Mendes, G. H. S.; Jugend, D. Fatores críticos de sucesso no gerenciamento de projetos de desenvolvimento de produto em empresas de base tecnológica de pequeno e médio porte. Gestão Produção, São Carlos, v. 15, n. 1, p. 117-134, jan.-abr. 2008.

Trot, Paul. Gestão da Inovação e Desenvolvimento de Novos Produtos. Porto Alegre. Bookman. 2012

Revista de Gestão e Projetos - GeP, São Paulo, v. 4, n. 3, p 119-140, set./dez. 2013. 
Zhu, J.; Cook; W. D. Data Envelopment Analysis: Modeling Operational Processes and Measuring Productivity. York University, Canada. Wade D. Cook.

Data do recebimento do artigo: 15/07/2013

Data do aceite de publicação: 02/09/2013

Revista de Gestão e Projetos - GeP, São Paulo, v. 4, n. 3, p 119-140, set./dez. 2013. 